\title{
Optical Properties of [all]-S,S-Dioxide Oligothiophenes
}

\author{
María M. Oliva, ${ }^{1, *}$ Sandra R. González, Juan Casado, \\ Juan T. López Navarrete, ${ }^{1}$ J. S. Seixas de Melo, ${ }^{2}$ Shlomo Rozen ${ }^{3}$ \\ ${ }^{1}$ Department of Physical Chemistry, University of Málaga, Málaga 29071, Spain \\ ${ }^{2}$ Department of Chemistry, University of Coimbra, Coimbra 3004-535, Portugal \\ ${ }^{3}$ School of Chemistry, Tel-Aviv University, Tel-Aviv 69978, Israel
}

Received 11 May 2009; accepted 18 May 2009

\begin{abstract}
A comprehensive photophysical and spectroscopic Raman study guided by Density Functional Theory and Hartree-Fock calculations has been done in a series of fully oxidized oligothiophenes with variable chain length, up to four rings. A comparison with the properties of oligoenes and oligothiophenes is proposed.
\end{abstract}

Keywords: oligothiophenes, $\pi$-conjugation, photophysics, Raman spectroscopy, theoretical calculations.

\section{Introduction}

Oligomers of thiophene are technologically important materials [1], constituting one of the most important family of molecules in the area of plastic electronics. Among their derivatives, the sulfone or $\mathrm{S}, \mathrm{S}^{\prime}$-dioxide derivatives, where the thienyl sulfur is oxidized to its dioxide state, have been intensively exploited. However, most of these studies have dealt with partially oxidized oligothiophenes since the synthetic difficulties to get the full sulfur oxidation. We want to check how sulfur atom oxidation of oligothiophenes, that decouples the pair of electrons of $\mathrm{S}$ from the thiophene ring aromaticity, alters their electronic and molecular structures. We propose a comparison between the nonoxidized species and their equivalent oligoenes (Fig. 1).

\footnotetext{
* Corresponding author. E-mail address: mmorenoo@uma.es
} 


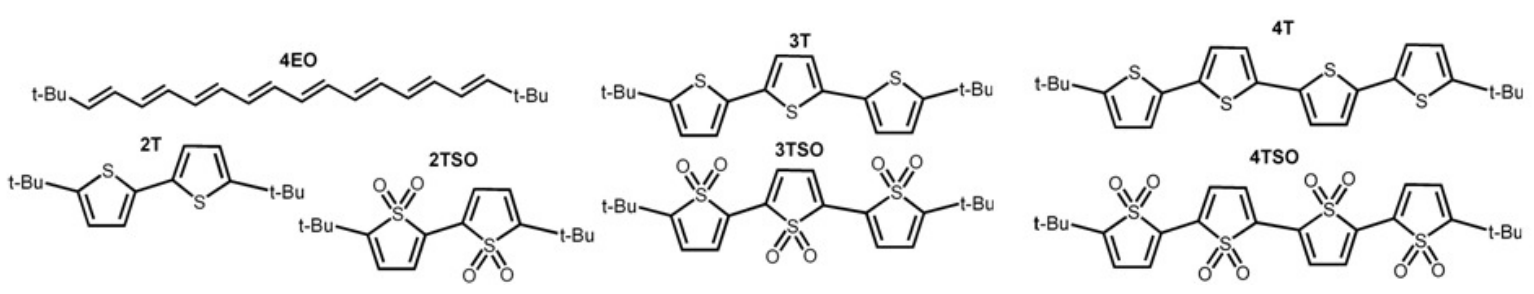

Figure 1. Chemical structures and acronyms of the compounds studied.

\section{Experimental and theoretical details}

The chemical synthesis of the studied compounds has been previously published [2]. The data presented in this paper for the oligoenes are those available in the literature [3]. UV-Vis absorption spectra were recorded on an Agilent 8453 instrument. Emission spectra were measured using a Spectrofluorimeter (FLS920P) from Edinburgh Analytical Instrument. Fluorescence quantum yields, $\phi_{\mathrm{F}}$, were measured using $1 \times 10^{-7} \mathrm{~mol} \mathrm{~L}^{-1}$ quinine sulfate in $0.1 \mathrm{~mol} \mathrm{~L}^{-1} \mathrm{H}_{2} \mathrm{SO}_{4}$ as the standard $\left(\phi_{\mathrm{F}}=0.546\right)$. FT-Raman scattering spectra were collected on a Bruker FRA106/S apparatus and a Nd:YAG laser source $\left(\lambda_{\text {exc }}=1064 \mathrm{~nm}\right)$. Raman spectra $\left(\lambda_{\text {exc }}=532 \mathrm{~nm}\right)$ were recorded by using a Senterra dispersive Raman microscope from Bruker. Theoretical data were calculated using Density Functional Theory by means of the Gaussian-03 package of programs [4].

\section{Results and discussion}

Fig. 2 displays the absorption and emission spectra of the compounds under study. The first observation is that fluorescence quantum yield decreases on going from the oligothiophenes to their dioxide derivatives. Very low fluorescence intensity is a property of oligoenes explained in terms of interference with a $2{ }^{1} \mathrm{Ag}$ singlet. Fluorescence emission of $2 \mathrm{~T}$ shows the typical vibronic features associated with the planarization of the $S_{1}$ state with intersection points of both spectra in the $0-0$ electronic origin meaning that the absorbing and emitting states are the same (i.e., small Stokes shifts). These observations contrast with those found in 2TSO and 3TSO since the luminescence spectra are featureless and largely displaced to the red.

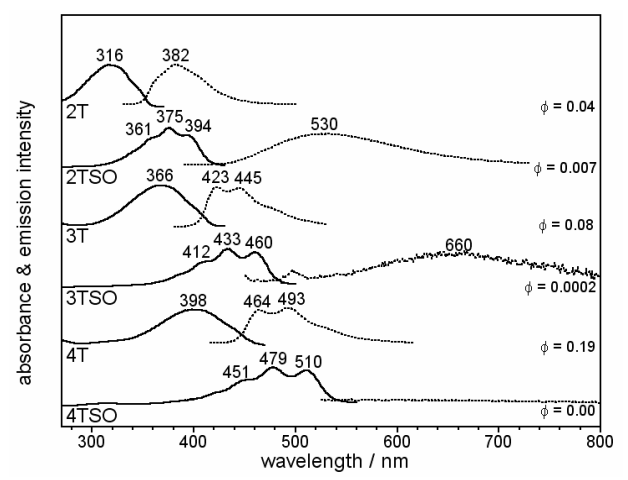

Figure 2. Absorption and emission spectra of the compounds under study at $25{ }^{\circ} \mathrm{C}$ in dichloromethane. 
As already mentioned, a decrease of the fluorescence emission upon going from the oligothiophenes to their dioxides is observed along with a further decrease of it on increasing the chain length, two findings that are possibly in agreement with the participation of an interfering dipole-forbidden non-emissive $2{ }^{1} \mathrm{Ag}\left(2{ }^{1} \mathrm{~A}_{1}\right)$ state close to the emissive $1{ }^{1} \mathrm{Bu}\left(1{ }^{1} \mathrm{~B}_{1}\right)$ in molecules with $\mathrm{C}_{2 \mathrm{~h}}\left(\mathrm{C}_{2 \mathrm{v}}\right)$ molecular symmetry. In Fig. 3 the TD-DFT data are summarized for the relevant excited states of 4TSO/4T showing the expected energy variation (i.e., stabilization) of the $2{ }^{1} \mathrm{Ag}$ state that would undergo by electron delocalization if we would take into account two-excitations (i.e., realize that these promotions are not considered in the DFT calculations).

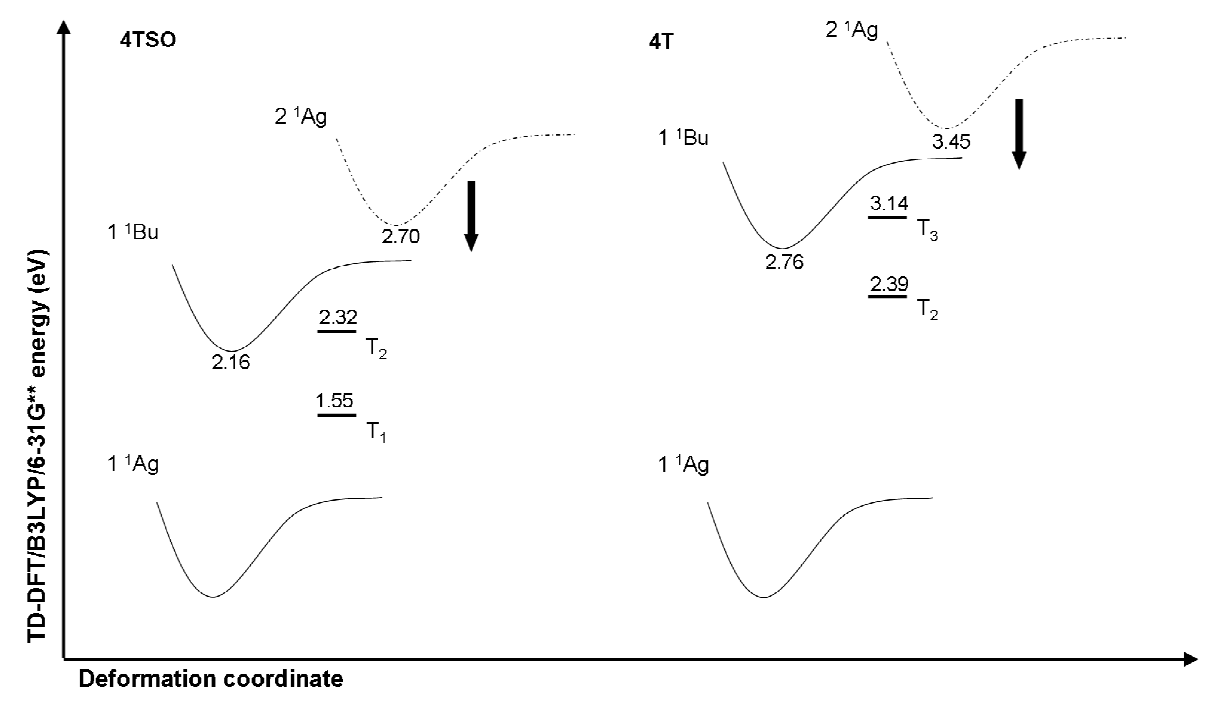

Figure 3. Energy diagram showing the singlet and triplet manifold according to TDDFT/B3LYP/6-31G** calculations in 4TSO (left) and in 4T (right). The effect of electron correlation is tried to be described producing a stabilization of the $2^{1} \mathrm{Ag}$ state (this energy would be likely predicted with CAS calculations).

Fig. 4 shows the FT-Raman spectra of 2OE, 2TSO and 2T in the solid state with an excitation wavelength of $1064 \mathrm{~nm}$. It is possible to correlate the Raman frequencies with the molecular structures of the $\mathrm{S}_{0}$ ground electronic state. The prominent bands in the three spectra are recorded at $1491 \mathrm{~cm}^{-1}$ in $2 \mathrm{~T}$, at 1545 $\mathrm{cm}^{-1}$ in $2 \mathrm{TSO}$, and at $1627 \mathrm{~cm}^{-1}$ in $2 \mathrm{OE}$. They are related to the $\mathrm{C}=\mathrm{C}$ stretching modes terminal of the rings in the bithiophene derivatives [5], and to the Ramanactive $v(C=C)$ mode in the oligoene. This assignment allows us to establish a structure-property relationship since the corresponding double bond is significantly shorter in $2 \mathrm{OE}$ in agreement with its larger vibrational frequency: $1491 \mathrm{~cm}^{-1}(2 \mathrm{~T}) \rightarrow 1545 \mathrm{~cm}^{-1}(2 \mathrm{TSO}) \rightarrow 1627 \mathrm{~cm}^{-1}(2 \mathrm{OE})$ and $1.375 \AA \mathrm{C}(2 \mathrm{~T}) \rightarrow$ $1.351 \AA(2 \mathrm{TSO}) \rightarrow 1.347 \AA$ (2OE).

Probably the simplification of the Raman spectrum in $2 \mathrm{OE}$ arises from a high degree of bond length equalization in the oligoene chain as a consequence of optimal $\pi$-electron conjugation. 

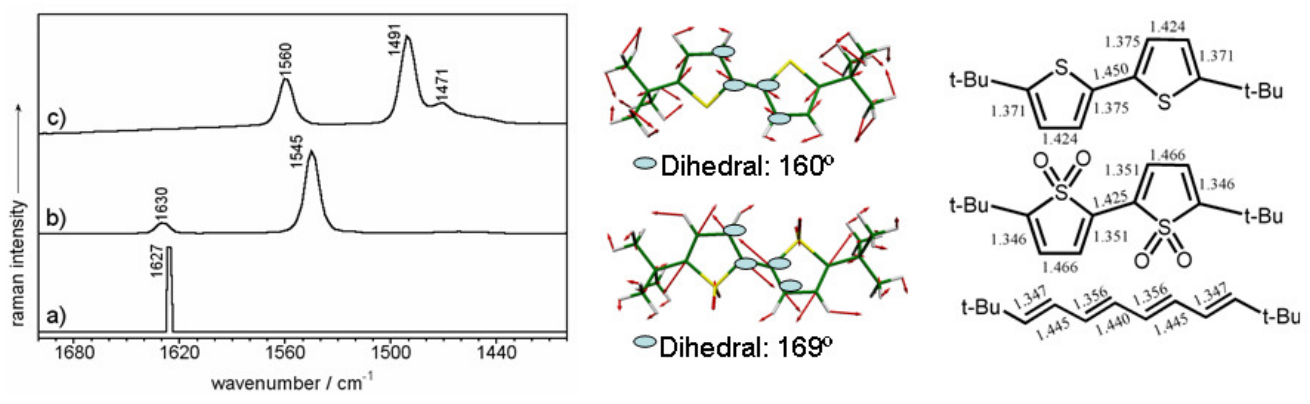

Figure 4. Left: Solid state Raman spectra of: a) $2 \mathrm{OE}$ represented as bars keeping the experimental relative intensities as in ref. 12, b) 2TSO, and c) 2T. Middle: DFT/B3LYP/6-31G** vibrational eigenvectors associated with the most important Raman line of 2TSO and 2T. Right: Ground electronic state DFT/B3LYP/6-31G** optimized geometries of 2OE, 2TSO and 2T.

From the beginning of conducting polymer research, it was realized that one of the main drawback of polyacetylene was its low ambient and thermal stability. We focus on the thermal stability of the dioxide oligothiophenes and their correspondence with oligoene and oligothiophenes. Fig. 5 shows the FT-Raman spectra of 4TSO in solid state in a large interval of temperatures. In the whole interval of temperature analyzed, the Raman spectra do not substantially change and only a gradual lack of Raman intensity is observed owing to sample heating. The spectra fully recover after thermal cycle indicating the strong resistance of the chemical attack or decomposition. This is a well known property of oligothiophenes which, among others, promoted their fast development and implementation in devices.

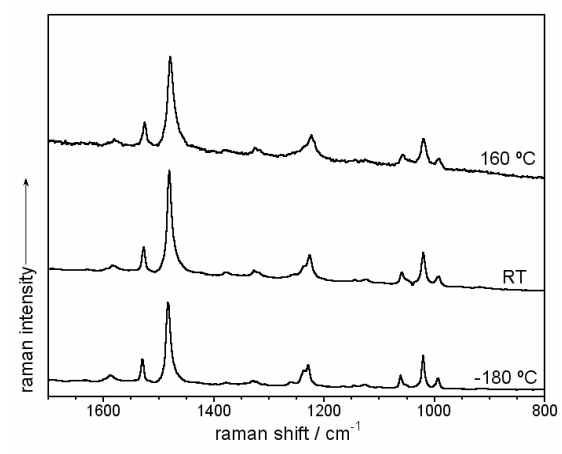

Figure 5. $532 \mathrm{~nm}$ FT-Raman spectra in solid state of 4TSO at high and low temperatures.

\section{Conclusions}

We have focused our interest in a series of fully oxidized oligothiophenes with variable chain length, viewed as oligothiophenelike and oligoenelike molecules. Their absorption and emission profiles contain vibronic peaks such as oligoenes. 
The same has been done for the structural properties on the basis of the Raman spectra which show a scenario of absence of thiophene aromaticity. Although the strategy of full sulfonation of oligothiophenes might represent an interesting organic synthesis protocol, it totally decouples the sulfur atoms from the thiophene aromaticity and thus minimizes the interesting emission properties of oligothiophenes. The full oxidation strategy should be followed to attain materials with lower optical band gap for solar cell applications, for example.

\section{References}

1. D. Fichou, Handbook of Oligo and Polythiophenes, Ed. Wiley-VCH: New York, 1999.

2. $\quad$ E. Amir, S. Rozen, Angew. Chem. Int. Ed. 44 (2005) 7374.

3. a) R.R. Chance, H. Schaffer, K. Knoll, R. Schrock, R. Silbey, Synth. Met. 49-50 (1992) 271.

b) K. Knoll, R. Schrock, J. Am. Chem. Soc. 111 (1989) 7989.

4. M.J. Frisch et al., Gaussian 03, Revision B.05, Gaussian, Inc.: Pittsburgh, PA, 2003.

5. a) J. Casado, H.E. Katz, V. Hernández, J.T. López Navarrete, J. Phys. Chem. B 106 (2002) 2488.

b) V. Hernández, J. Casado, F.J. Ramírez, G. Zotti, S. Hotta, J.T. López Navarrete, J. Chem. Phys. 104 (1996) 9271. 